\title{
Evaluating Diagnostic Accuracy and Determining Optimal Diagnostic Thresholds of Three Different Approaches to [68Ga]-DOTATATE PET/MRI Analysis in Patients with Meningioma
}

\author{
Sean Kim \\ Weill Cornell Medicine \\ Michelle Roytman \\ Weill Cornell Medicine \\ Gabriela Madera \\ Weill Cornell Medicine \\ Rajiv Magge \\ Weill Cornell Medicine \\ Benjamin Liechty \\ Weill Cornell Medicine \\ Rohan Ramakrishna \\ Weill Cornell Medicine \\ Susan Pannullo \\ Weill Cornell Medicine \\ Theodore Schwartz \\ Weill Cornell Medicine \\ Nicolas Karakatsanis \\ Weill Cornell Medicine \\ Joseph Osborne \\ Weill Cornell Medicine \\ Eaton Lin \\ Weill Cornell Medicine \\ Jonathan Knisely \\ Weill Cornell Medicine \\ Jana Ivanidze ( $\square$ jai9018@med.cornell.edu ) \\ Weill Cornell Medicine
}

Research Article 
Keywords: DOTATATE, PET/MRI, meningioma, SUV, diagnostic threshold

Posted Date: December 1st, 2021

DOI: https://doi.org/10.21203/rs.3.rs-1096920/v1

License: (c) (1) This work is licensed under a Creative Commons Attribution 4.0 International License. Read Full License 


\section{Abstract}

\section{PURPOSE}

Multiple approaches with [Ga68]-DOTATATE, a somatostatin analog PET radiotracer, have demonstrated clinical utility in evaluation of meningioma but have not been compared directly. Our purpose was to compare diagnostic performance of three approaches to quantitative brain [68Ga]-DOTATATE PET/MRI analysis in patients with suspected meningioma recurrence and to establish the optimal diagnostic threshold for each method.

\section{METHODS}

Patients with suspected meningioma were imaged prospectively with [68Ga]-DOTATATE brain PET/MRI. Lesions were classified as meningiomas and post-treatment change (PTC), based on pathology findings and follow up MRI appearance. Lesions were reclassified using the following methods: absolute SUV threshold (SUV), SUV ratio (SUVR) to superior sagittal sinus (SSS) (SUVRsss), and SUVR to the pituitary gland (SUVRpit). Diagnostic performance of the three methods was compared using contingency tables and McNemar's test. Previously published pre-determined thresholds were assessed where applicable. The optimal thresholds for each method were identified using Youden's J statistics.

\section{RESULTS}

166 meningiomas and 41 PTC lesions were identified across 62 patients. SUV, SUVRsss, and SUVRpit of meningioma were significantly higher than those of PTC $(P<0.0001)$. The optimal thresholds for SUV, SUVRsss, and SUVRpit were 4.65, 3.23, and 0.260, respectively. At the optimal thresholds, SUV had the highest specificity (97.6\%) and SUVRsss had the highest sensitivity (86.1\%). An ROC analysis of SUV, SUVRsss, and SUVRpit revealed AUC of $0.932,0.910$, and 0.915 , respectively $(P<0.0001)$.

\section{CONCLUSION}

We found that the SUVRsss method may have the most robust combination of sensitivity and specificity in the diagnosis of meningioma in the post-treatment setting, with the optimal threshold of 3.23. Future studies validating our findings in different patient populations are needed to continue optimizing the diagnostic performance of [68Ga]-DOTATATE PET/MRI in meningioma patients.

Clinical Trial Registration: ClinicalTrials.gov Identifier: NCT04081701. Registered 9 September 2019. https://clinicaltrials.gov/ct2/show/NCT04081701

\section{Introduction}

Meningiomas are the most common primary intracranial tumors, accounting for more than a third of all primary brain tumors [1]. While gross-total resection is the standard of care, approximately $34-50 \%$ of 
patients undergo subtotal resection which is associated with lower rates of progression-free survival (PFS) and overall survival (OS), necessitating subsequent active surveillance with serial imaging for detection of any residual or recurrent tumor [2]. In patients with high-risk meningioma, which include newly diagnosed or recurrent WHO grade 3 meningioma of any resection extent, a recurrent WHO grade 2 tumor of any resection extent, or a newly diagnosed WHO grade 2 tumor following subtotal resection, postoperative adjuvant radiotherapy (RT) is often pursued [3]. Magnetic resonance imaging (MRI) is the gold standard for the diagnosis and treatment planning of meningioma. However, especially in the postsurgical and post-RT setting, MRI can have suboptimal sensitivity and specificity in distinguishing meningioma from post-treatment scarring and inflammation [4]. MRI is also limited in cases of small lesion size, infiltrative or "en plaque" lesions, osseous or parenchymal invasion, and challenging locations such as the skull base and cavernous sinus [5-7]. Thus, more sensitive and specific imaging biomarkers have the potential to improve diagnosis, treatment, and thereby clinical outcomes in meningioma.

[Ga68]-DOTATATE is a positron emission tomography (PET) radiotracer that targets somatostatin receptor 2 (SSTR2), which is highly expressed in meningiomas, with greater affinity than other somatostatin receptor analogs [8,9]. Since achieving the orphan drug status in 2014 by the Food and Drug Administration, [68Ga]-DOTATATE PET has proven to be superior to other functional imaging modalities in meningioma such as Indium-111-Octreotide scintigraphic imaging, demonstrating improved specificity and target-to-background ratio [10]. [68Ga]-DOTATATE PET has also demonstrated its superior clinical utility in target volume delineation during radiation planning, in the detection of transosseous, small, or difficultly located meningiomas, and in post-surgical settings, when compared to contrast enhanced MRI alone [4-7, 11].

However, while highly specific to meningioma, the level of [68Ga]-DOTATATE avidity in meningioma that constitutes the optimal diagnostic threshold has not been systematically investigated. Furthermore, the specificity of [68Ga]-DOTATATE avidity in meningioma is affected by the variable physiologic uptake in other tissues including the pituitary, salivary, thyroid glands, liver, spleen, and urinary tract [12]. Several different methods have been utilized in previous studies to classify lesions as meningioma on the basis of [68Ga]-DOTATATE PET when interpreting standard uptake value (SUV) in meningioma, often with reference to SUV in a background tissue, such as contralateral brain parenchyma, contralateral subarachnoid space, liver, gluteal muscle, and superior sagittal sinus [4, 5, 13-15]. While the liver reference recapitulates the Krenning score, first established on the basis of Indium-111-Octreotide scintigraphic imaging, it does not reflect the differences in receptor specificity between [68Ga]-DOTATATE and Octreotide. Importantly, it requires whole-body PET in addition to brain PET, which confers longer acquisition time, thereby affecting patient comfort as well as cost. Additionally, the potential for incidental findings on the whole-body MRI may lead to undue patient anxiety and unwarranted workups [16]. Thus, approaches focusing on data obtained from dedicated brain acquisition alone hold promise in optimizing the acquisition and analysis protocols while maintaining patient comfort and limiting acquisition time. 
Published diagnostic approaches include an absolute maximum SUV threshold of 2.3, which demonstrated $90 \%$ sensitivity and $73 \%$ specificity for the purpose of diagnosing meningioma from tumor free tissue, which have been utilized in several other studies $[5,17,18]$. For surgical and radiation planning purposes, however, which demand a higher confidence level for diagnosis and delineation of a tumor, a greater specificity may be desired. Another previously published method uses the superior sagittal sinus (SSS) as a reference, given its role as cranial blood pool, with a proposed SUV ratio (SUVR) of 3 to distinguish meningioma from post-treatment change [4]. Our purpose was to compare the three different approaches to determining optimal diagnostic thresholds using [68Ga]-DOTATATE PET/MR in the differentiation of meningioma versus post-treatment change in the post-surgical setting, including the absolute SUV threshold of 2.3, the SUVR to SSS with the threshold of 3, and the SUVR to the pituitary gland, an intracranial organ with consistently high physiologic [68Ga]-DOTATATE avidity, and to establish the optimal threshold SUV values for each method with the greatest sensitivity and specificity.

\section{Methods}

\section{Patient Population}

Institutional review board approval with informed consent was obtained for this retrospective HIPAA compliant study of patients with a history of clinically suspected or pathology-proven meningioma. A total of 88 [68Ga]-DOTATATE PET/MRI examinations were obtained between July 2018 and March 2021 as part of a prospective clinical trial for the purpose of diagnosing meningioma or differentiating recurrence from post-treatment change. Five patients who were ineligible for PET/MRI underwent PET/CT and MRI separately. From this cohort, all patients with imaging evidence of meningioma and posttreatment changes were included. All patients with a diagnosis other than meningioma were excluded (Figure 1). In patients with multiple longitudinal [68Ga]-DOTATATE PET/MRI examinations, only the initial scan was included. Clinical chart review was performed to collect clinical and demographic characteristics of the study population, including age, sex, surgical history, radiation treatment history, as well as the number of meningiomas in each patient. All methods were carried out in accordance with relevant guidelines and regulations.

\section{Image Acquisition}

PET/MRI was performed on the Biograph mMR scanner (Siemens Healthineers, Erlangen, Germany) or the GE SIGNATM PET/MR scanner (GE Healthcare, Milwaukee, WI). PET imaging started immediately post-injection of approximately 5 millicuries of [68Ga]-DOTATATE. PET data were acquired in 3D List Mode for a total of 50 minutes. MRI was performed according to institutional protocol, including pre- and postcontrast sagittal 3D T1 SPACE (TR/TE 600-700 ms/11-19 ms, 120 degree flip, 1 mm slice thickness) and 3D T2 FLAIR (TR/TE 6300-8500 ms/394-446 ms, 120 degree flip, 1 mm slice thickness). MR-based attenuation correction was obtained according to manufacturer's standard-of-care specifications. For patients who underwent PET/CT and MRI separately, the CT image set of the PET/CT was subsequently registered to the postcontrast $\mathrm{T} 1$-weighted $\mathrm{MR}$ images using the rigid registration algorithm residing on 
Syngo.ViaTM workstation (Siemens Healthineers, Erlangen, Germany) and the resulting transformation matrix was then applied to the PET image set to register it to the MRI images.

\section{Quantitative Imaging Analysis}

Maximum SUV of the meningiomas and post-treatment change lesions were calculated based on the injected dose of the radiotracer and patient body weight. The lesions were confirmed as meningioma versus post-treatment change based on the pathology findings, if available, and follow up MR imaging appearance. In patients with multiple meningiomas, only the lesions that were directly biopsied or surgically removed were categorized as pathology proven. In the absence of pathology and imaging follow up, the current gold standard MRI appearance from the [68Ga]-DOTATATE PET/MR was used to classify the lesions. MRI based classification of meningioma was based upon prototypical imaging characteristics including well-circumscribed margins, lobular morphology, avid contrast enhancement, and extra-axial location with a broad-based dural attachment, often with an associated dural tail, as determined by both the interpreting neuroradiologist and secondary review from study neuroradiologists. Other characteristics that were considered ancillary evidence of a meningioma diagnosis included internal areas of calcification and inward displacement of adjacent cortex.

Any indeterminate lesions were excluded. Any previously irradiated radiation treated residual meningiomas were also excluded. The breakdown of the number of lesions confirmed by each of the above criteria is outlined in Figure 1. The lesions that were confirmed as meningiomas or post-treatment changes were then reclassified using the following three methods: an absolute SUV threshold of 2.3 as described in reference [17], an SUV ratio (SUVR) referencing the superior sagittal sinus (SSS) threshold of 3 as described in reference [4], and an SUVR referencing the pituitary gland (SUVRpit). Given that no previously published reference exists for the SUVRpit approach, the optimal threshold for this method was determined by performing Youden's $\mathrm{J}$ statistics within our cohort.

\section{Statistical Analysis}

Mann-Whitney test was performed to compare SUV, SUVRsss, and SUVRpit values between meningioma and post-treatment changes. Kruskal-Wallis test was performed to compare SUV, SUVRsss, and SUVRpit values between WHO grades in meningioma. In patients with multiple meningiomas, given that many simultaneously occurring meningiomas show a uniform histology, all meningiomas, including those that were not directly biopsied, were assigned the same WHO grade within a given patient, following the methodology applied in prior studies [19-21]. In order to evaluate potential bias when analyzing multiple meningiomas per patient, an additional evaluation with only pathology proven meningiomas was performed. Linear regression analysis was performed to correlate SUV, SUVRsss, and SUVRpit with the size of meningioma as determined by the length of the longest dimension in a subset of patients whose meningioma size information was available. Diagnostic accuracy of the three different classification methods was assessed using $2 \times 2$ contingency tables and sensitivity, specificity, positive predictive value (PPV), and negative predictive value (NPV) were calculated for each method. The statistical difference between the three diagnostic methods was evaluated using the McNemar test. Receiver-operating- 
characteristic analyses were performed to compare the diagnostic performance of SUV, SUVRsS, and SUVRpit using area under the curve. Youden's $\mathrm{J}$ statistics was performed to identify the optimal thresholds in each method. At the identified optimal thresholds, the McNemar test was again used to compare the three diagnostic methods. GraphPad Prism 8 was used to perform all statistical analyses. P values below 0.05 were considered to indicate statistical significance.

\section{Results}

\section{Study Population}

Of the initial cohort of 88 [68Ga]-DOTATATE PET/MRI and PET/CT, 3 scans with diagnosis other than meningioma and 13 scans that were repeated follow up scans of the same patient were excluded. In total, 62 patients met the inclusion criteria (Figure 1). Among the 214 lesions identified on [68Ga]DOTATATE PET/MR in 62 patients, 3 indeterminate lesions in the absence of pathology and imaging follow ups and 4 lesions that were post-radiation treated residual tumors were excluded. In total, 166 meningiomas and 41 post-treatment change lesions were identified across the cohort. Of the 166 meningiomas, $16 \%(26 / 166)$ were confirmed with pathology outcome, with the median time from PET to pathology of 8 days (range: 1 day-1.4 years) (Figure 1). 4.9\% (2/41) of the post treatment change lesions were confirmed with pathology, with median time from PET to pathology of 8 days. $77 \%(128 / 166)$ of meningiomas and $78 \%(32 / 41)$ of post-treatment change were confirmed with follow up MRI, with the median time from PET to follow up of 8.6 months for meningioma (1.8 months-2.5 years) and of 13.2 months for post-treatment change (4 days-2.5 years). Finally, $7.2 \%(12 / 166)$ of meningiomas and $17 \%$ (7/41) of post treatment change were confirmed on the basis of the MR appearance at the time of [68Ga]DOTATATE PET/MR. Detailed clinical and demographic characteristics of the study population are outlined in Table 1. A representative patient images from the cohort is shown in Figure 2 and 3.

Table 1 Demographic characteristics of the study population 


\begin{tabular}{|c|c|}
\hline \# Patients & 62 \\
\hline $\begin{array}{l}\text { \# Patients with a History of Pathology Proven } \\
\text { Meningioma }\end{array}$ & $87 \%(54 / 62)$ \\
\hline Age & $55.9(21-89)$ \\
\hline Sex & $66 \% \mathrm{~F}(41 / 62)$ \\
\hline Surgical History & $85 \%(53 / 62)$ \\
\hline Time from Surgery to PET & Median: 8.0 months (19 days -14.6 yrs) \\
\hline Radiation History & $23 \%(14 / 62)$ \\
\hline Time from Radiation to PET & $\begin{array}{l}\text { Median: } 27.6 \text { months (10 months - } 17.3 \\
\text { yrs) }\end{array}$ \\
\hline N Meningiomas & 166 \\
\hline N Post-Treatment Changes & 41 \\
\hline \multirow[t]{5}{*}{ N Meningiomas per patient } & $15 \%(9 / 62)$ with 0 meningioma \\
\hline & $35 \%(22 / 62)$ with 1 meningioma \\
\hline & $34 \%(21 / 62)$ with 2-4 meningiomas \\
\hline & $16 \%(10 / 62)$ with $>4$ meningiomas \\
\hline & Median: 1.5 meningiomas per scan $(0-16)$ \\
\hline \multirow[t]{4}{*}{ N Post-Treatment Change Lesions per patient } & $53 \%(33 / 62)$ with 0 post-tx change \\
\hline & $32 \%(20 / 62)$ with 1 post-tx change \\
\hline & $15 \%(9 / 62)$ with $2-4$ post-tx changes \\
\hline & Median: 0 post-tx change per scan (0-4) \\
\hline \multirow[t]{4}{*}{ WHO grade } & $31 \%(19 / 62)$ WHO grade 1 \\
\hline & $47 \%(29 / 62)$ WHO grade 2 \\
\hline & $10 \%(6 / 62)$ WHO grade 3 \\
\hline & $13 \%(8 / 62)$ WHO grade not available \\
\hline
\end{tabular}

\section{Descriptive and Correlative Analysis of SUV}

Mean and range of SUV in meningioma and post-treatment change lesions as well as in the pituitary gland and SSS of the cohort are outlined in Table 2. Mean SUV of meningioma was significantly higher than that of post-treatment change lesions (15.8 vs. 2.58, P<0.0001) (Figure 4). Youdon's J statistics revealed 0.260 as the optimal threshold for SUVRpit. Mean SUVRsss and SUVRpit of meningioma were also significantly higher than that of post-treatment change lesions ( 11.5 vs. $2.10, P<0.0001 ; 0.92$ vs. 
$0.16, P<0.0001$, respectively) (Figure 4). There was no correlation between WHO grade and SUV, SUVRss, or SUVRpit of meningioma in the cohort $(\mathrm{P}=0.23, \mathrm{P}=0.56, \mathrm{P}=0.23$, respectively) (Figure 5). The additional analysis with the lesions that are pathology proven confirmed this relationship. Regression analysis revealed lesion size as a significant predictor of all SUV, SUVRsss, and SUVRpit in meningioma $(P<0.005$, $R 2=0.066 ; P<0.0005, R 2=0.096 ; P=0.0014, R 2=0.079$, respectively).

Table 2 Descriptive analysis of the target lesions. Only a subset $(n=9)$ of the post-treatment change lesions had its size information available

\begin{tabular}{|ll|}
\hline & Mean (Range) \\
\hline Pituitary SUV & $16.7(7-34.2)$ \\
\hline SSS SUV & $1.39(0.6-2.8)$ \\
\hline Meningioma SUV & $15.8(1.1-111.8)$ \\
\hline Meningioma SUVRss & $11.5(0.52-136.1)$ \\
\hline Meningioma SUVRpit & $0.92(0.065-10.3)$ \\
\hline Meningioma Size (n=126) (cm) & $1.34(0.2-4.7)$ \\
\hline Post-Tx Change SUV & $2.58(0-8.5)$ \\
\hline Post-Tx Change SUVRss & $2.1(0-5.5)$ \\
\hline Post-Tx Change SUVRpit & $0.16(0-0.39)$ \\
\hline Post-Tx Change Size $(\mathrm{n}=9)(\mathrm{cm})$ & $0.9(0.3-1.6)$ \\
\hline
\end{tabular}

\section{Diagnostic Performance of the Three Classification Methods at the Pre-Determined Thresholds}

Contingency tables for the three classification methods are shown in Tables 3, 4, and 5. Sensitivity, specificity, PPV, and NPV of the three methods are compared in Table 6, along with the prevalence of meningioma in the cohort. At the pre-determined thresholds, SUV demonstrated the highest sensitivity (98.2\%) and the highest specificity was achieved with SUVRpit (87.8\%) (Table 6). As shown in Table 7, the sensitivity of the SUV method was significantly greater than the sensitivities of both SUVRsss and SUVRpit methods $(P<0.0001)$. However, the specificity of the SUV method was significantly lower than that of both SUVRsss and SUVRpit ( $P=0.0044$ and $P=0.0009$, respectively). The sensitivity of SUVRsss was significantly higher than that of SUVRpit $(P=0.006)$ while the specificities of both SUVR methods did not differ significantly $(P=0.3711)$.

Table 3 Contingency table for the SUV method at the pre-determined threshold of 2.3 


\begin{tabular}{|llll|}
\hline$>2.3$ SUV & Meningioma & Post-Tx Change & Total \\
\hline Positive & 163 & 18 & 181 \\
\hline Negative & 3 & 23 & 26 \\
\hline Total & 166 & 41 & 207 \\
\hline
\end{tabular}

Table 4 Contingency table for the SUVRpit method at the threshold of 0.260

\begin{tabular}{|llll|}
\hline$>0.260$ SUVRpit & Meningioma & Post-Tx Change & Total \\
\hline Positive & 132 & 5 & 137 \\
\hline Negative & 34 & 36 & 70 \\
\hline Total & 166 & 41 & 207 \\
\hline
\end{tabular}

Table 5 Contingency table for the SUVRsss method at the pre-determined threshold of 3

\begin{tabular}{|llll|}
\hline$>$ 3 SUVRss & Meningioma & Post-Tx Change & Total \\
\hline Positive & 144 & 8 & 152 \\
\hline Negative & 22 & 33 & 55 \\
\hline Total & 166 & 41 & 207 \\
\hline
\end{tabular}

Table 6 Sensitivities, specificities, positive predictive value (PPV), negative predictive value (NPV) of the three methods with $95 \%$ confidence interval at the pre-determined thresholds

\begin{tabular}{|llll|}
\hline & $>2.3$ SUVmax & $>0.260$ SUVRpit & $>3$ SUVRss \\
\hline Sensitivity & $98.2 \%(94.8-99.5)$ & $79.5 \%(72.7-85.0)$ & $86.7 \%(80.8-91.1)$ \\
\hline Specificity & $56.1 \%(41.0-70.1)$ & $87.8 \%(74.5-94.7)$ & $80.5 \%(66.0-89.8)$ \\
\hline PPV & $90.1 \%(84.8-93.6)$ & $96.4 \%(91.7-98.4)$ & $94.7 \%(90.0-97.3)$ \\
\hline NPV & $88.5 \%(71.0-96.0)$ & $51.4 \%(40.0-62.8)$ & $60.0 \%(46.8-71.9)$ \\
\hline Prevalence & $80.2 \%(166 / 207)$ & $80.2 \%(166 / 207)$ & $80.2 \%(166 / 207)$ \\
\hline
\end{tabular}

Table 7 McNemar's Test Results comparing the three methods at the pre-determined thresholds

\begin{tabular}{|llll|}
\hline & SUV vs SUVRpit & SUVRpit vs SUVRsss & SUV vs SUVRsss \\
\hline Sensitivity & $\mathrm{P}<0.0001^{*}$ & $\mathrm{P}=0.0060^{*}$ & $\mathrm{P}<0.0001^{*}$ \\
\hline Specificity & $\mathrm{P}=0.0009 *$ & $\mathrm{P}=0.3711$ & $\mathrm{P}=0.0044^{\star}$ \\
\hline
\end{tabular}




\section{Diagnostic Performance of the Three Classification Methods at Their Optimal Thresholds}

Table 8 shows the optimal thresholds for each of the three methods as determined by Youdon's $\mathrm{J}$ statistics and the sensitivities and specificities for each method at the optimal thresholds. Of note, the optimal threshold for SUVRsss method was 3.23, similar to the threshold of 3 as used in the previous study [4]. The optimal threshold for SUV was 4.65 in our cohort, greater than the pre-determined threshold of 2.3. At the optimal thresholds, SUV had the highest specificity (97.6\%) and SUVRsss had the highest sensitivity (86.1\%). The sensitivity of SUV was significantly lower than that of SUVRsss while the specificity of SUV was significantly higher than that of SUVRsss ( $P=0.0012$ and $P=0.0412$, respectively) (Table 9). The SUVRpit method was not statistically different from the SUV method in both sensitivity and specificity, however it had a significantly lower sensitivity than the SUVRsss method ( $P=0.0098)$ with no significant difference in specificities. A receiver-operating-characteristic analysis of SUV, SUVRsss, and SUVRpit as diagnostic parameters for meningioma revealed area under curve of $0.932(P<0.0001), 0.910$ $(P<0.0001)$, and $0.915(P<0.0001)$, respectively (Figure 6).

Table 8 Optimal threshold of each method as determined by Youden's J Statistics with 95\% confidence interval

\begin{tabular}{|lllll|}
\hline Methods & J & Optimal Threshold & Sensitivity & Specificity \\
\hline SUV & 75.27 & $>4.65$ & $77.7 \%(70.8-83.4)$ & $97.6 \%(87.4-99.9)$ \\
\hline SUVRsss & 69.07 & $>3.227$ & $86.1 \%(80.1-90.6)$ & $82.9 \%(68.7-91.5)$ \\
\hline SUVRpituitary & 67.32 & $>0.260$ & $79.5 \%(72.7-85.0)$ & $87.8 \%(74.5-94.7)$ \\
\hline
\end{tabular}

Table 9 The McNemar's Test for sensitivity/specificity of the three methods at the optimal thresholds

\begin{tabular}{|llll|}
\hline & SUV vs SUVRpit & SUVRpit vs SUVRss & SUV vs SUVRss \\
\hline Sensitivity & $\mathrm{P}=0.505$ & $\mathrm{P}=0.0098^{\star}$ & $\mathrm{P}=0.0012^{\star}$ \\
\hline Specificity & $\mathrm{P}=0.1336$ & $\mathrm{P}=0.6171$ & $\mathrm{P}=0.0412^{\star}$ \\
\hline
\end{tabular}

\section{Discussion}

Recurrence is relatively common in meningioma patients post-treatment, occurring in up to $20 \%$ of cases, even in histologically benign (WHO grade 1) cases [22]. In order to minimize the rate of recurrence as well as the side effects from excessive treatment to normal brain tissue, an ideal imaging modality should be specific enough to define the correct tumor volume from tumor-free tissue, thereby aiding in surgical and radiation planning to achieve the maximal safe target volume. In the post-treatment setting, it should also be sensitive to detect any residual or recurrent tumor from post-treatment scarring and inflammation. The most recently updated European Association of Neuro-Oncology guideline for the management of meningioma continues to highlight contrast-enhanced MRI as the gold standard for diagnosing and 
follow up imaging in meningioma patients [23]. However, MRI appearance is often limited in accuracy particularly in the presence of post-treatment scarring and inflammation. Rachinger and colleagues demonstrated that standard MRI has sensitivity of $79 \%$ and specificity of $65 \%$ in distinguishing meningioma from tumor-free tissues [17]. Another group showed that MRI alone can achieve sensitivity of $95 \%$ and specificity $88 \%$ in the diagnosis of meningioma in a cohort of 57 patients, but noted that diagnostic accuracy diminishes in cases of small lesions $\left(<0.5 \mathrm{~cm}^{3}\right)$ and certain difficult locations such as skull base [6]. For transosseous growing meningiomas, MRI's sensitivity was even lower at $54 \%$ [5].

The recently updated European Association of Neuro-Oncology guidelines also highlight the role of [68Ga]-DOTATATE PET in distinguishing meningioma from healthy tissue and post-surgical changes [23]. Histology-controlled studies showed that the extent of meningiomas is better delineated with [68Ga]DOTATATE PET compared to contrast-enhanced MRI alone $[5,17]$. During radiation planning, [68Ga]DOTATATE and DOTATOC PET alter target volume delineation for stereotactic fractionated radiation therapy, often resulting in a reduction of the gross tumor volume compared with results from MRI or CT $[11,24,25]$. The utility of adjuvant RT, compared to active surveillance, in resected meningiomas is currently being evaluated in the NRG0539 trial, in which our preliminary analysis suggests [68Ga]DOTATATE's efficacy for RT response assessment with a marked reduction in [68Ga]-DOTATATE SUV in meningioma post-RT. Additionally, a case series of 20 patients demonstrated the clinical utility of [68Ga]DOTATATE PET/MR in identifying additional meningiomas not previously identified on contrast-enhanced $\mathrm{MRI}$ and in differentiating disease from reactive enhancement, thus facilitating treatment planning in such cases [4]. Further reinforcing its clinical utility, [68Ga]-DOTATATE PET has shown efficacy in predicting progression in non-benign meningioma as well as predicting clinical outcome for SSTR targeted radionuclide therapy such as Lu-DOTATATE $[18,19]$. Within our cohort, we confirmed that all 3 approaches to [68Ga]-DOTATATE PET quantification (absolute SUV, SUVRsss and SUVRpit) aid in the differentiation of meningioma from post-treatment changes, confirming the reliability of the SSTR2 targeted imaging in meningioma patients. SUV, SUVRsss, and SUVRpit did not correlate with WHO grade, consistent with prior histopathological studies, and suggesting that SSTR2 expression is independent of the differentiation status of meningioma tumor cells $[17,26]$. There was a significant relationship observed between tumor size and SUV, SUVRsss, and SUVRpit.

In order to effectively utilize [68Ga]-DOTATATE PET in the clinical context of meningioma, an SUV threshold that constitutes as the diagnostic threshold must be established. Prior studies often employed reference tissue SUVR approaches, such as contralateral brain parenchyma, contralateral subarachnoid space, liver, gluteal muscle, and superior sagittal sinus [4, 5, 13-15]. One prospective study of 21 patients reported absolute SUV threshold of 2.3 with $90 \%$ sensitivity and $73 \%$ specificity for the purpose of diagnosing meningioma from tumor free tissue, which have been utilized in several other studies $[5,17$, 18]. One other well-known method is the Krenning score system, derived from [111 In]Octreotide scintigraphy of gastrointestinal neuroendocrine tumors, which uses the liver and spleen as the reference regions and has been validated in neuroendocrine tumors that are SSTR2 positive for the purpose of assessing candidacy for PRRT [27]. However, the Krenning score has not been utilized in meningioma and 
requires a body PET. Therefore, we evaluated PET/MRI-based approaches to quantitative [68Ga]DOTATATE PET analysis that do not require whole-body imaging, and instead rely on dynamic brain PET imaging. Amongst the anatomic regions that can be obtained from brain PET, we chose the SSS or cranial blood pool as a background reference region, as previously published, and the pituitary gland, a notably SSTR2 positive intracranial organ [4].

In our cohort of 62 patients, at the pre-determined threshold, the SUV method with the threshold of 2.3 demonstrated the highest sensitivity (98.2\%) but much lower specificity than the prior study of 21 patients (56.1\% vs. $73.5 \%$ ) [5]. The optimal threshold for SUV in our cohort was 4.65 , much greater than the threshold of 2.3 in the prior study [5]. This discrepancy may be explained by the different sample sizes, varying acquisition time and technicality of imaging, and random variation in SUV across patients. The highest specificity was achieved with SUVRpit (87.8\%) with the threshold of 0.260 but it was not significantly different from the specificity of SUVRsss at the pre-determined threshold of 3 , which had a greater sensitivity than SUVRpit. We then recalculated sensitivity and specificity of each classification method at the optimal threshold as determined by Youden's $\mathrm{J}$ statistics. Notably, the optimal threshold for SUVRsss was 3.23 , similar to 3 as set by the prior study and as tested in our analysis of the predetermined thresholds [4]. Interestingly, at the optimal thresholds, the methods with the highest sensitivities and specificities were flipped; SUV had the highest specificity (97.6\%) and SUVRss had the highest sensitivity (86.1\%). At the optimal thresholds, the SUVRpit method was not statistically different from the SUV method, however it had a significantly lower sensitivity than the SUVRsss method with no difference in specificities, suggesting that SUVRsss is a superior method overall compared to SUVRpit. AUCs of the ROC for each method were comparable. Based on this result, we conclude that SUVRsss with the threshold of 3.23 may be used in clinical settings where greater sensitivity is desired such as in the post treatment setting in the evaluation of recurrence or progression. The SUV threshold of 4.65 may be more appropriate for instances where high specificity is desired such as surgical and radiation planning. However, it is important to note that SUVR that is normalized to a region of interest in the same patient may be more reproducible and thus a more reliable metric of SSTR expression, allowing more robust comparison across time points, scanners, and patients. Furthermore, given greater variability of SUV of the pituitary gland compared to SUV of the SSS, SUVRsss may be the more robust of the two SUVR methods and thus the preferred method in the clinical setting.

Our study has several limitations. It is important to note that a significant number of individual lesions included in the study (77\% of meningiomas) were confirmed based on clinical follow ups rather than biopsy. For the purpose of correlating WHO grades and SUV in meningioma, multiple meningiomas in a given patient were assigned the same WHO grade as the WHO grade of the pathology proven meningioma in the given patient, as applied in previously published studies [19-21]. Additionally, the number of the pathology proven post-treatment change is relatively low given that they are most often confirmed with longitudinal follow ups with MRI as the gold standard. Notably, $17 \%$ of the post-treatment change lesions included in the study were confirmed with the MR appearance of the [68Ga]-DOTATATE $\mathrm{PET} / \mathrm{MR}$ in the absence of both biopsy and clinical follow ups, which may affect the accuracy of the classification. Additionally, while we excluded meningiomas that were previously irradiated from our 
analysis, recurrence in prior RT fields might affect SUV of the lesions when compared with de novo or untreated meningiomas, an important issue which we plan to study in future work. Finally, a SSTR2 negative meningioma, although exceedingly rare, may contribute to potential misclassification of the lesions [28].

\section{Conclusion}

[68Ga]-DOTATATE PET/MR has emerged in recent years as a useful adjunct modality for management of meningioma in various clinical contexts, including diagnosis, treatment planning and response assessment, and recurrence surveillance. Our study represents a systematic comparison of quantitative analysis approaches to differentiating meningioma from post-treatment change. We compared absolute SUV as well as SUVR thresholds in their diagnostic performance in diagnosing meningioma in the post treatment setting and determined the optimal numerical threshold values that achieve the greatest sensitivity and specificity. Our analysis reveals that the SUVRsss approach with an optimal threshold of 3.23 achieves the greatest sensitivity while the absolute SUV threshold of 4.65 has the highest specificity. Recognizing that the SUVR method may be a more reproducible metric, the SUVRsss method may convey the greatest clinical utility. Further studies that investigate our threshold values in cohorts of varying sizes and compositions are required to validate our findings in other clinical contexts and assess the effect of demographic factors on SSTR biology in meningioma. Continued efforts to standardize interpretation and diagnostic criteria of [68Ga]-DOTATATE PET/MR imaging in meningioma have the potential of improving diagnosis and treatment and thereby improve clinical outcomes for patients with meningioma.

\section{Abbreviations}

PFS (progression free survival), OS (overall survival), RT (radiation treatment), MRI (magnetic resonance imaging), SSTR2 (somatostatin receptor 2), PET (positron emission tomography), SUV (standard uptake value), SSS (superior sagittal sinus), SUVR (standard uptake value ratio), SUVRpit (standard uptake value ratio relative to pituitary gland), SUVRsss (standard uptake value ratio relative to superior sagittal sinus), PPV (positive predictive value), NPV (negative predictive value), WHO (world health organization).

\section{Declarations}

\section{Ethics approval and consent to participate}

Institutional review board approval with informed consent was obtained from the ethics committee of Weill Cornell Medicine for this retrospective HIPAA compliant study.

\section{Consent for publication}

Consent to publish any individual data has been obtained as part of the informed consent process.

\section{Availability of data and material}


The datasets used and/or analyzed during the current study are derived from an ongoing clinical trial and are available from the corresponding author on reasonable request.

\section{Competing interests}

The authors declare that they have no competing interests that are relevant to the content of this manuscript.

\section{Funding}

This work was supported by Advanced Accelerator Applications, A Novartis Company [Study Number: CAAA501A0US05T] as part of the DOMINO-START clinical trial protocol [ClinicalTrials.gov Identifier: NCT04081701].

\section{Authors' contributions}

Conceptualization: Jana Ivanidze, Sean H. Kim; Methodology: Jana Ivanidze, Michelle Roytman, Sean H. Kim; Formal analysis and investigation: Jana Ivanidze, Michelle Roytman, Sean H. Kim; Writing - original draft preparation: Jana Ivanidze, Sean $\mathrm{H}$. Kim, Writing - reviewing and editing: all authors; Enrollment: Sean H. Kim, Michelle Roytman, Gabriela Madera, Rajiv Magge, Rohan Ramakrishna, Susan C. Pannullo, Theodore H. Schwartz, Joseph R. Osborne, Eaton Lin, Jonathan P.S. Knisely, Jana Ivanidze; Patient management: Sean H. Kim, Michelle Roytman, Gabriela Madera, Rajiv Magge, Rohan Ramakrishna, Susan C. Pannullo, Theodore H. Schwartz, Joseph R. Osborne, Eaton Lin, Jonathan P.S. Knisely, Jana Ivanidze; PET/MR protocol optimization: Jana Ivanidze, Nicolas Karakatsanis, Sean H. Kim; Pathology review: Benjamin Liechty; PET/MR analysis: Jana Ivanidze, Michelle Roytman, Eaton Lin, Sean H. Kim; Final approval of the manuscript: all authors

\section{Acknowledgements}

Not applicable.

\section{References}

1. Ostrom QT, Gittleman H, Liao P, Vecchione-Koval T, Wolinsky Y, Kruchko C, et al. CBTRUS Statistical Report: Primary brain and other central nervous system tumors diagnosed in the United States in 2010-2014. Neuro Oncol. 2017;19:v1-v88. doi:10.1093/neuonc/nox158.

2. Rogers $L$, Barani I, Chamberlain M, Kaley TJ, McDermott M, Raizer J, et al. Meningiomas: knowledge base, treatment outcomes, and uncertainties. A RANO review. J Neurosurg. 2015;122:4-23. doi:10.3171/2014.7.JNS131644.

3. Wang D, Sun S, Hua L, Deng J, Luan S, Cheng H, et al. Prognostic Model That Predicts Benefits of Adjuvant Radiotherapy in Patients With High Grade Meningioma. Front Oncol. 2020;10:568079-. doi:10.3389/fonc.2020.568079. 
4. Ivanidze J, Roytman M, Lin E, Magge RS, Pisapia DJ, Liechty B, et al. Gallium-68 DOTATATE PET in the Evaluation of Intracranial Meningiomas. Journal of Neuroimaging. 2019;29:650-6. doi:https://doi.org/10.1111/jon.12632.

5. Kunz WG, Jungblut LM, Kazmierczak PM, Vettermann FJ, Bollenbacher A, Tonn JC, et al. Improved Detection of Transosseous Meningiomas Using (68)Ga-DOTATATE PET/CT Compared with ContrastEnhanced MRI. J Nucl Med. 2017;58:1580-7. doi:10.2967/jnumed.117.191932.

6. Einhellig HC, Siebert E, Bauknecht H-C, Tietze A, Graef J, Furth C, et al. Comparison of diagnostic value of $68 \mathrm{Ga}$-DOTATOC PET/MRI and standalone MRI for the detection of intracranial meningiomas. Scientific Reports. 2021;11:9064. doi:10.1038/s41598-021-87866-9.

7. Nyuyki F, Plotkin M, Graf R, Michel R, Steffen I, Denecke T, et al. Potential impact of 68Ga-DOTATOC $\mathrm{PET} / \mathrm{CT}$ on stereotactic radiotherapy planning of meningiomas. European Journal of Nuclear Medicine and Molecular Imaging. 2010;37:310-8. doi:10.1007/s00259-009-1270-2.

8. Reubi JC, Schär JC, Waser B, Wenger S, Heppeler A, Schmitt JS, et al. Affinity profiles for human somatostatin receptor subtypes SST1-SST5 of somatostatin radiotracers selected for scintigraphic and radiotherapeutic use. Eur J Nucl Med. 2000;27:273-82. doi:10.1007/s002590050034.

9. Silva CB, Ongaratti BR, Trott G, Haag T, Ferreira NP, Leães CG, et al. Expression of somatostatin receptors (SSTR1-SSTR5) in meningiomas and its clinicopathological significance. Int J Clin Exp Pathol. 2015;8:13185-92.

10. Gabriel M, Decristoforo C, Kendler D, Dobrozemsky G, Heute D, Uprimny C, et al. \&lt;sup\&gt;68\&lt;/sup\&gt;Ga-DOTA-Tyr\&lt;sup\&gt;3\&lt;/sup\&gt;-Octreotide PET in Neuroendocrine Tumors: Comparison with Somatostatin Receptor Scintigraphy and CT. Journal of Nuclear Medicine. 2007;48:508. doi:10.2967/jnumed.106.035667.

11. Mahase SS, Roth O'Brien DA, No D, Roytman M, Skafida ME, Lin E, et al. [(68)Ga]-DOTATATE $\mathrm{PET} / \mathrm{MRI}$ as an adjunct imaging modality for radiation treatment planning of meningiomas. Neurooncol Adv. 2021;3:vdab012. doi:10.1093/noajnl/vdab012.

12. Hofman MS, Lau WFE, Hicks RJ. Somatostatin Receptor Imaging with 68Ga DOTATATE PET/CT: Clinical Utility, Normal Patterns, Pearls, and Pitfalls in Interpretation. RadioGraphics. 2015;35:500-16. doi:10.1148/rg.352140164.

13. Afshar-Oromieh A, Giesel FL, Linhart HG, Haberkorn U, Haufe S, Combs SE, et al. Detection of cranial meningiomas: comparison of 68Ga-DOTATOC PET/CT and contrast-enhanced MRI. European Journal of Nuclear Medicine and Molecular Imaging. 2012;39:1409-15. doi:10.1007/s00259-0122155-3.

14. Parghane RV, Talole S, Basu S. Prevalence of hitherto unknown brain meningioma detected on (68)Ga-DOTATATE positron-emission tomography/computed tomography in patients with metastatic neuroendocrine tumor and exploring potential of (177)Lu-DOTATATE peptide receptor radionuclide therapy as single-shot treatment approach targeting both tumors. World J Nucl Med. 2019;18:160-70. doi:10.4103/wjnm.WJNM_39_18. 
15. Pelak MJ, d'Amico A. The Prognostic Value of Pretreatment Gallium-68 DOTATATE Positron Emission Tomography/Computed Tomography in Irradiated Non-benign Meningioma. Indian J Nucl Med. 2019;34:278-83. doi:10.4103/ijnm.IJNM_98_19.

16. Schmidt CO, Sierocinski E, Hegenscheid K, Baumeister SE, Grabe HJ, Völzke H. Impact of whole-body MRI in a general population study. European Journal of Epidemiology. 2016;31:31-9. doi:10.1007/s10654-015-0101-y.

17. Rachinger W, Stoecklein VM, Terpolilli NA, Haug AR, Ertl L, Pöschl J, et al. Increased $<$ sup $>68<$ /sup>Ga-DOTATATE Uptake in PET Imaging Discriminates Meningioma and Tumor-Free Tissue. Journal of Nuclear Medicine. 2015;56:347-53. doi:10.2967/jnumed.114.149120.

18. Seystahl K, Stoecklein V, Schüller U, Rushing E, Nicolas G, Schäfer N, et al. Somatostatin receptortargeted radionuclide therapy for progressive meningioma: benefit linked to 68Ga-DOTATATE/-TOC uptake. Neuro Oncol. 2016;18:1538-47. doi:10.1093/neuonc/now060.

19. Sommerauer M, Burkhardt JK, Frontzek K, Rushing E, Buck A, Krayenbuehl N, et al. 68GalliumDOTATATE PET in meningioma: A reliable predictor of tumor growth rate? Neuro Oncol. 2016;18:1021-7. doi:10.1093/neuonc/now001.

20. Mocker K, Holland H, Ahnert P, Schober R, Bauer M, Kirsten H, et al. Multiple meningioma with different grades of malignancy: case report with genetic analysis applying single-nucleotide polymorphism array and classical cytogenetics. Pathol Res Pract. 2011;207:67-72. doi:10.1016/j.prp.2010.09.001.

21. Butti G, Assietti R, Casalone R, Paoletti P. Multiple meningiomas: a clinical, surgical, and cytogenetic analysis. Surg Neurol. 1989;31:255-60. doi:10.1016/0090-3019(89)90048-7.

22. Maillo A, Orfao A, Sayagues JM, Diaz P, Gómez-Moreta JA, Caballero M, et al. New classification scheme for the prognostic stratification of meningioma on the basis of chromosome 14 abnormalities, patient age, and tumor histopathology. J Clin Oncol. 2003;21:3285-95. doi:10.1200/jco.2003.07.156.

23. Goldbrunner R, Stavrinou P, Jenkinson MD, Sahm F, Mawrin C, Weber DC, et al. EANO guideline on the diagnosis and management of meningiomas. Neuro Oncol. 2021. doi:10.1093/neuonc/noab150.

24. Milker-Zabel S, Zabel-du Bois A, Henze M, Huber P, Schulz-Ertner D, Hoess A, et al. Improved target volume definition for fractionated stereotactic radiotherapy in patients with intracranial meningiomas by correlation of CT, MRI, and [68Ga]-DOTATOC-PET. Int J Radiat Oncol Biol Phys. 2006;65:222-7. doi:10.1016/j.jjrobp.2005.12.006.

25. Graf R, Nyuyki F, Steffen IG, Michel R, Fahdt D, Wust P, et al. Contribution of 68Ga-DOTATOC PET/CT to target volume delineation of skull base meningiomas treated with stereotactic radiation therapy. Int J Radiat Oncol Biol Phys. 2013;85:68-73. doi:10.1016/j.ijrobp.2012.03.021.

26. Durand A, Champier J, Jouvet A, Labrousse F, Honnorat J, Guyotat J, et al. Expression of c-Myc, neurofibromatosis Type 2, somatostatin receptor 2 and erb-B2 in human meningiomas: relation to grades or histotypes. Clin Neuropathol. 2008;27:334-45. doi:10.5414/npp27334. 
27. Krenning EP, Valkema R, Kooij PP, Breeman WA, Bakker WH, deHerder WW, et al. Scintigraphy and radionuclide therapy with [indium-111-labelled-diethyl triamine penta-acetic acid-D-Phe1]-octreotide. Ital J Gastroenterol Hepatol. 1999;31 Suppl 2:S219-23.

28. Roytman M, Pisapia DJ, Liechty B, Lin E, Skafida M, Magge RS, et al. Somatostatin receptor-2 negative meningioma: pathologic correlation and imaging implications. Clinical Imaging. 2020;66:18-22. doi:https://doi.org/10.1016/j.clinimag.2020.04.026.

\section{Figures}


88 [68Ga]-DOTATATEPET (83

PET/MR + 5 PET/CT)

Excluded 3 scans with diagnosis other than meningioma +13 repeated follow up scans of the same patient

62 unique patients reviewed

Excluded 3 indeterminate lesions +4 postradiation treated residual tumors

Total 207 lesions included (166 meningiomas +41 post-treatment change)

Confirmed with pathology

- $16 \%(26 / 166)$ meningiomas. Median time from PET to pathology: 8 days (1 day- 1.4 years)

- $4.9 \%(2 / 41)$ post-treatment change. Median time from PET to pathology: 8 days ( $8-8$ days)

Confirmed with follow up MRI

- $77 \%(128 / 166)$ meningiomas. Median time from PET to follow up: 8.6 months ( 1.8 months- 2.5 years)

- $78 \%(32 / 41)$ post-treatment change. Median time from PET to follow up: 13.2 months ( 4 days- 2.5 years)

Confirmed with MR appearance at the time of [68Ga]-DOTATATE PET/MR

- $7.2 \%(12 / 166)$ meningiomas

- $17 \%(7 / 41)$ post-treatment change

\section{Figure 1}

Flow chart of the included subjects and lesions as confirmed by each diagnostic criterion 

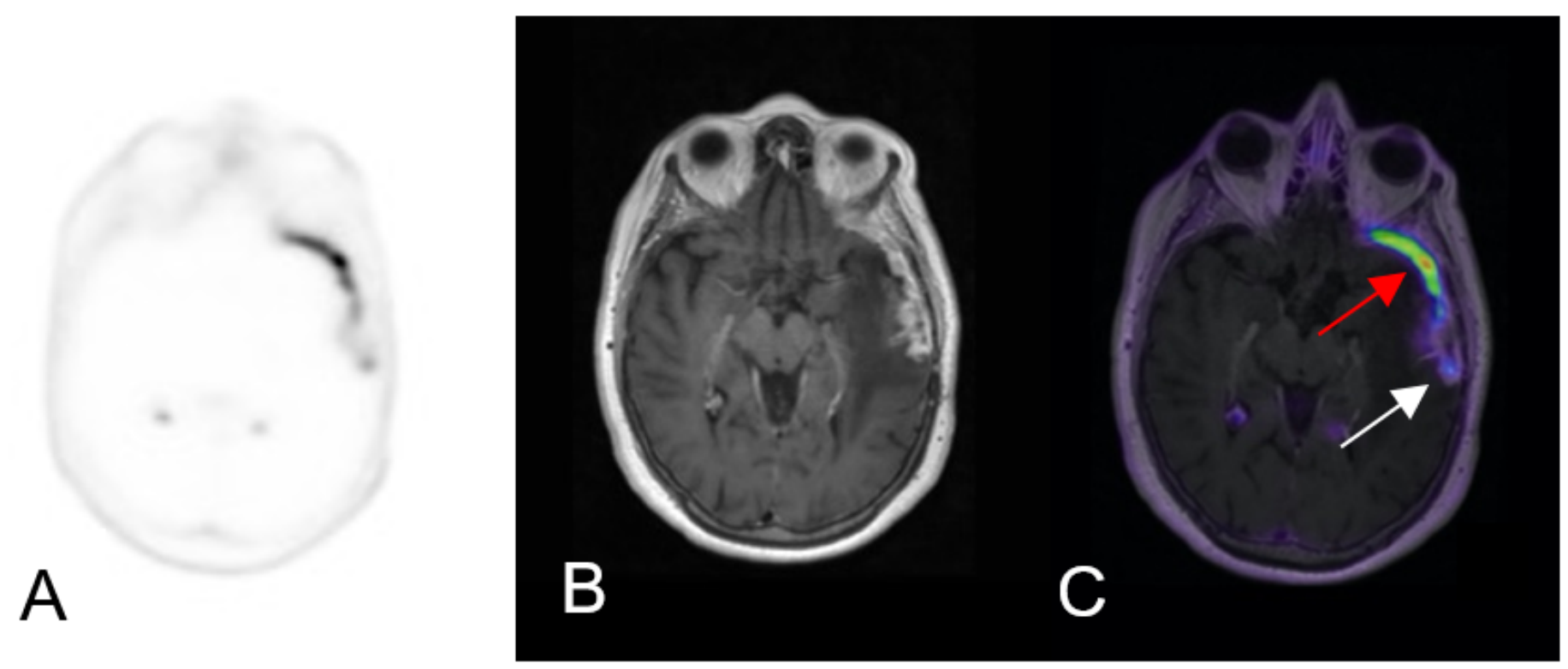

\section{Figure 2}

Axial images of [68Ga]-DOTATATE PET AC (A), 3D T1 post-gadolinium MR (B), fused PET/MR T1 (C) windowed SUV 0-15. This representative patient from the cohort is a 70-year-old female with a history of WHO grade II meningioma s/p resection and proton therapy 6 years prior to imaging and an additional proton therapy a year prior. The fused PET/MR images demonstrate a lesion suspicious for a meningioma in the left anterior temporal pole with SUV of 13.6 (C, red arrow). The more posteriorly located enhancing lesion (C, white arrow) demonstrates SUV of 4.5 and was suspicious for posttreatment change, given that the SUV of the superior sagittal sinus was 2.3. The subsequent resection and biopsy of the two lesions a year later confirmed the suspected diagnosis of recurrent meningioma and radiation necrosis, respectively 


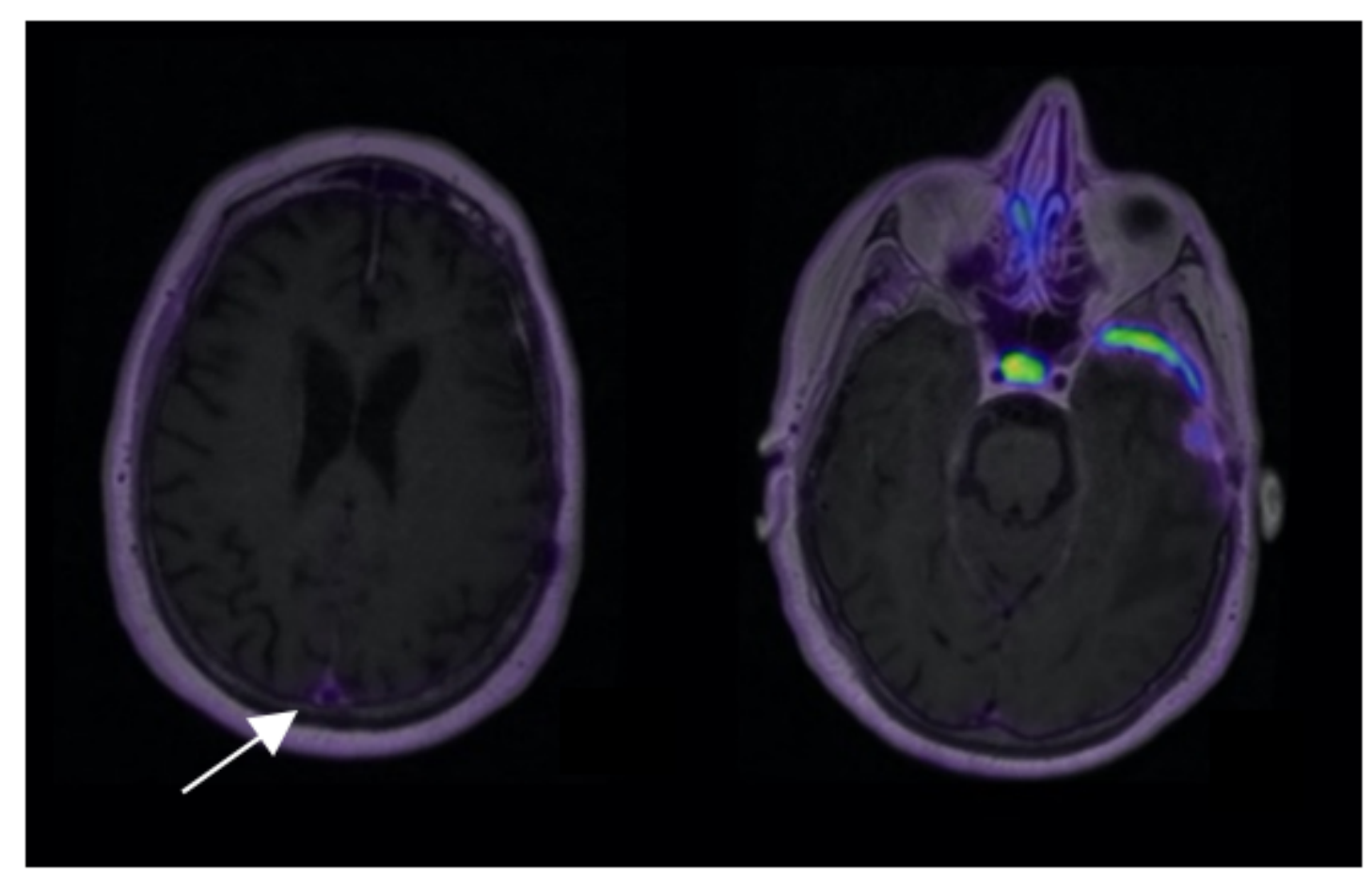

\section{Figure 3}

Axial images of fused PET/MR T1 windowed SUV 0-15 of the same representative patient from Figure 2. The superior sagittal sinus (left, arrow) demonstrates SUV of 2.3 and the pituitary gland (right) demonstrates SUV of 12.4
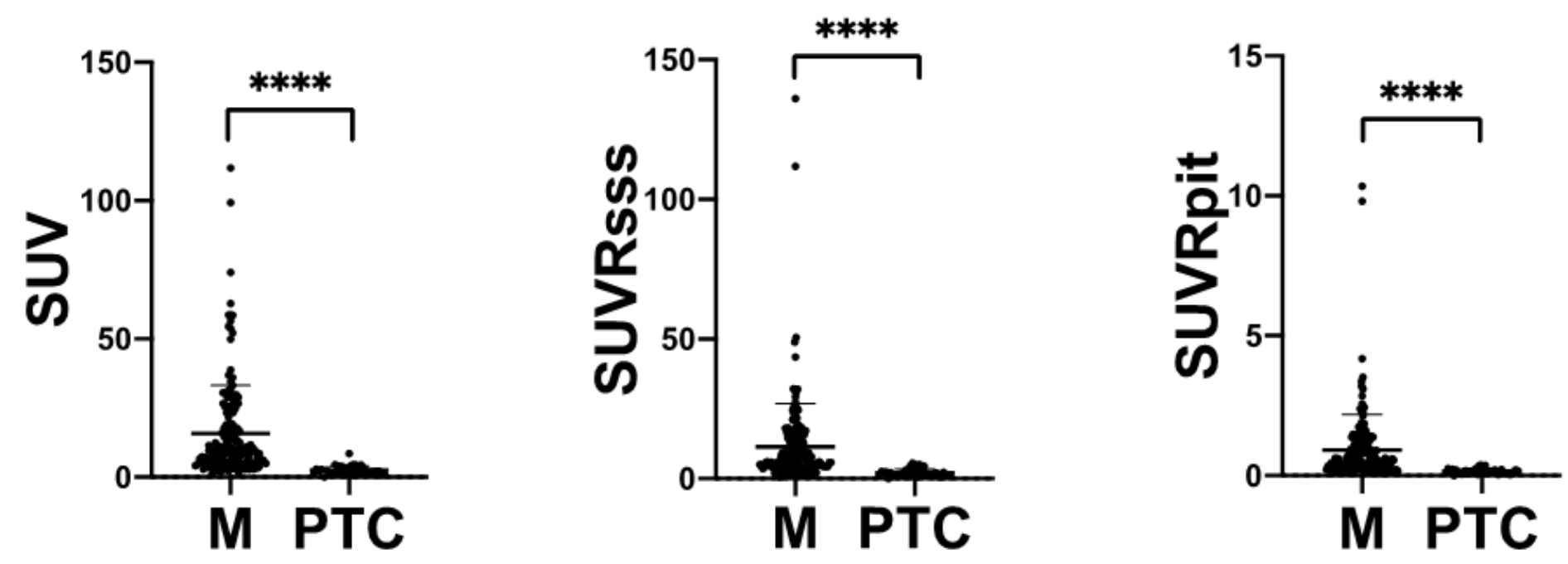

Figure 4

Meningioma (M) versus Post-treatment change (PTC) SUV (left), SUVRsss (middle), SUVRpit (right) with mean and standard deviations. Mean SUV, SUVRsss, and SUVRpit of meningioma was 15.8, 11.5, and 0.92 , respectively. ${ }^{\star \star \star \star}$ indicates statistical significance with $p<0.0001$ as determined by Mann-Whitney test 

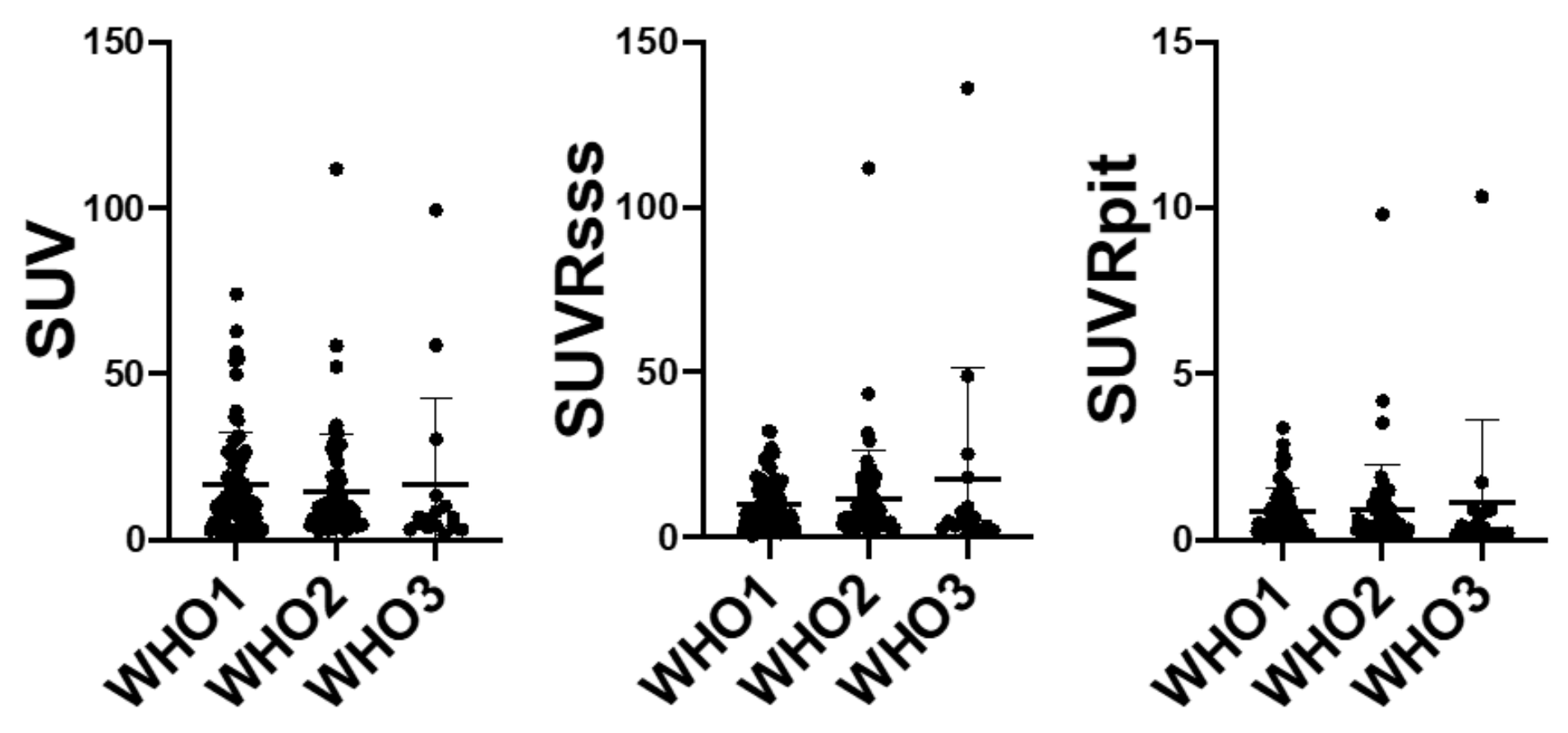

Figure 5

Meningioma SUV (left), SUVRsss (middle), and SUVRpit (right) stratified by WHO Grades with mean and standard deviations. Kruskal-Wallis test showed no statistically significant relationship between WHO Grade and the three SUV metrics

\section{ROC Curves}

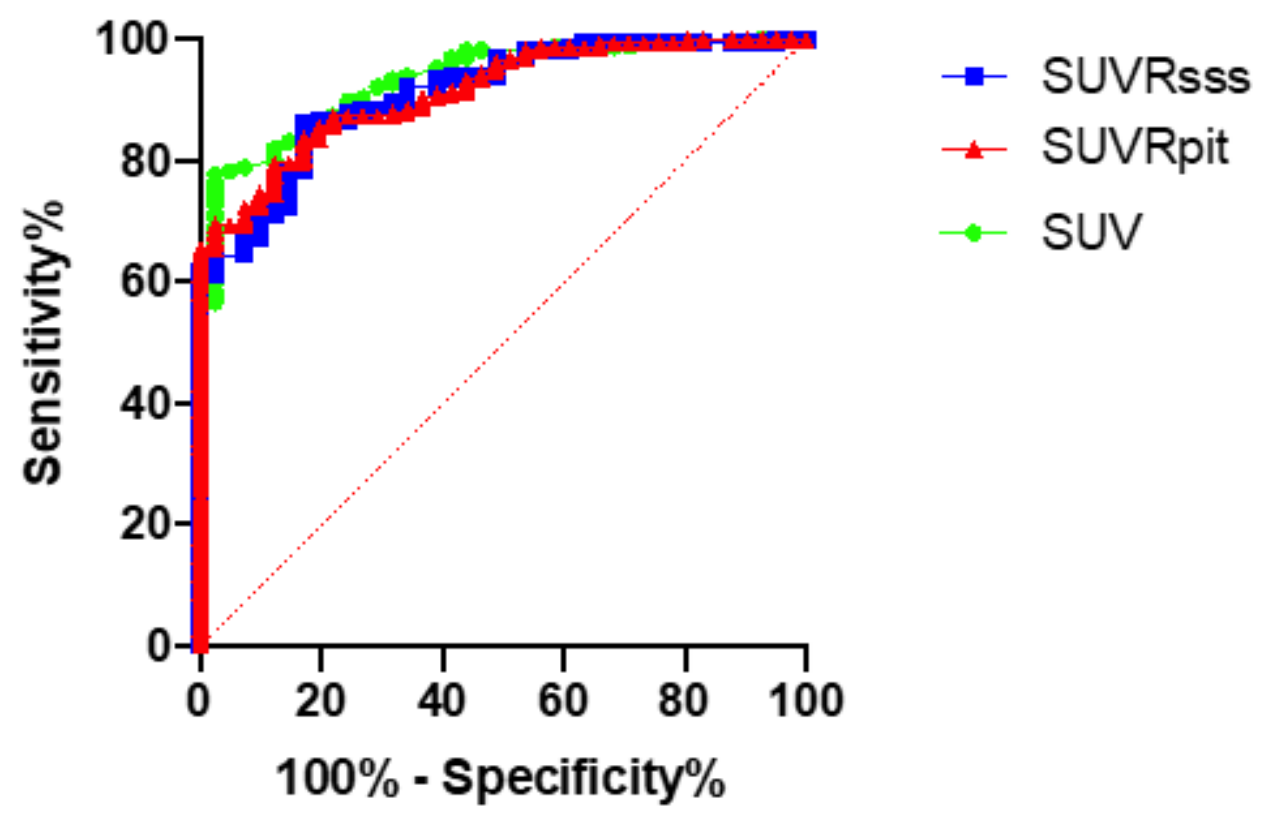

Figure 6 
ROC Curves of the three methods: SUV, SUVRsss, and SUVRpit. ROC analysis revealed area under the curve of $0.932,0.910$, and 0.915 for SUV, SUVRsss, and SUVRpit, respectively (all $P<0.0001$ ) 